Sharif University of Technology
Scientia Iranica
SCIENTIA

\title{
A meshless method to simulate interactions between large soft tissue and a surgical grasper
}

\author{
Z. Saghaei Nooshabadi ${ }^{a}$, E. Abdi ${ }^{b}$, F. Farahmand ${ }^{a}$, R. Narimani ${ }^{a}$ \\ and M. Chizari $i^{a, c, *}$ \\ a. School of Mechanical Engineering, Sharif University of Technology, Tehran, P.O. Box 11155-9567, Iran. \\ b. Institute of Microengineering, EPFL, CH-1015 Lausanne, Switzerland. \\ c. Orthopaedic Research and Learning Centre, College of Engineering, Design and Physical Sciences, Brunel University London, \\ Uxbridge, UB8 $3 P H, U K$.
}

Received 28 October 2014; received in revised form 30 December 2014; accepted 5 May 2015

\section{KEYWORDS \\ Intra-abdominal; \\ Large deformation; \\ Finite element; \\ Meshless method.}

\begin{abstract}
Realistic simulation of tool-tissue interactions can help to develop more effective surgical training systems and simulators. This study uses a finite element and meshless modeling approach to simulate the grasping procedure of a large intra-abdominal organ, i.e. a kidney, during laparoscopic surgery. Results indicate that the accuracy of the meshless method is comparable with that of the finite element method, with root mean square errors in the range of 0.8 to $2.3 \mathrm{~mm}$ in different directions. For the model presented in this study, the computational cost of the meshless method was much less than that of the finite element model.

(C) 2016 Sharif University of Technology. All rights reserved.
\end{abstract}

\section{Introduction}

Laparoscopic surgery is a keyhole surgical or minimally invasive surgical approach that has achieved an increasing popularity in clinical practice in recent years. In contrast to open surgery that needs large areas of cutting, laparoscopic surgery requires only small perforation holes for the entry of optical and surgical instruments into the body. With this type of surgery, the damage to the surrounding healthy tissue is minimum, which results in less infection and postoperative pain. However, this type of surgery requires that surgeons be trained for blindsurgery, which requires undertaking operations under a different environment. Most importantly, laparoscopic surgeons

\footnotetext{
*. Corresponding author. Tel.: +98 (21) 66165583 Fax: +98 (21) 6600021

E-mail addresses: zeynab_saghaee@yahoo.com (Z. Saghaei

Nooshabadi); elahe.abdi@epfl.ch (E.Abdi);

farahmand@sharif.edu (F.Farahmand);

narimani@sharif.edu (R. Narimani);

mahmoudchizari@yahoo.com (M. Chizari)
}

miss their direct vision and sense of touch over the operating organs, as they watch the surgery site using an optic system on a screen and perform surgery using long rod endoscopic instruments. So, it is necessary to provide new methods for laparoscopic surgery trainees that can enhance their skills in time and cost efficient ways. Surgical simulation systems provide virtual environments wherein trainees can practice surgery repeatedly at minimal cost.

Biomechanical modelling of soft tissue is a method to simulate and compute force-displacement interactions between the laparoscopic tool and the tissue. The method can provide the visual and haptic feedback needed for training. Detailed modelling of biological organs is complex, but it is essential to model such organs and, in order to model the biomechanical behavior of the organ, appropriate modelling tools are needed. Although experimental study is a choice for modelling the organs, complicated techniques may be required to measure the force-displacement characteristics experimentally [1]. There have been attempts in the literature to simulate the mechanical interac- 
tion of surgical tools and soft organs using different modeling techniques, e.g. Finite Element Method (FEM) [2-4], mass spring [5], and meshless method [6]. FEM is a common method in modelling soft tissue, but it is computationally more expensive than the meshless method and thus not appropriate for real time simulations. The meshless method, on the other hand, is not as accurate as FEM, but is much faster. It is also suitable for real time simulations, due to the fact that it does not need a mesh generation procedure. This is particularly important for tissue with complex geometry, as well as for easy handling of finite strains and large displacements in a Lagrangian framework [7].

The aim of this study is to compare the efficacy of FEM and meshless techniques used for fast simulation of tool-tissue interactions. For a soft large organ, mechanical integration with a laparoscopic grasper was simulated, and the accuracy and computational cost of the two techniques were compared.

\section{Method}

\subsection{Geometry of the kidney}

To simulate interaction between a kidney and a laparoscopic grasper, the three dimensional (3D) geometry of the kidney and grasper has to be measured. To measure the geometry of the kidney, a dummy model of the kidney was produced using a mold making and casting technique, as shown in Figure 1(a). Polyurethane foam liquid, as instructed in open literature (http://www.brickintheyard.com/), was used to make the model. Possible shrinkage of the foam model during the molding process was neglected. The foam model (Figure 1(b)) was then measured using a digital vernier caliper. All measurement records were then used to create a 3D geometrical model of the kidney in SolidWorks (DassaultSystèmes, Fr).

The size of the kidney model was about $30 \times$ $40 \times 75 \mathrm{~mm}$. A measurement was also taken from a custom made grasper, and its 3D model was created in SolidWorks software.

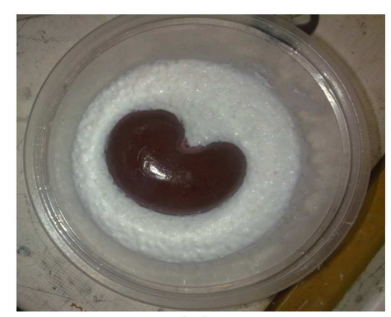

(a)

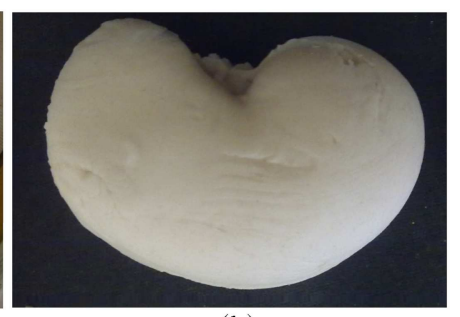

(b)
Figure 1. (a) A dummy model of the kidney produced by using a mold made of polyurethane foam liquid. (b) The geometry of the kidney extracted from the solid foam model. Small shrinkage of the foam model during the molding process was neglected.

\subsection{Material property}

For a clear comparison between the FEM and meshless models, identical mechanical properties were defined for both. To simplify the models, the mechanical properties of the kidney model were assumed to be an isotropic, homogeneous, incompressible material with linear elastic properties. The material properties of the soft tissue kidney were obtained from open literature [8]. The density and Young's modulus of the kidney were assumed to be $1000 \mathrm{~kg} / \mathrm{m}^{3}$ and $20 \mathrm{kPa}$, respectively, and its Poisson ratio to be 0.495 , considering the nearly incompressible behavior of the tissue.

No material was defined for the surgical grasper in the Finite Element (FE) model as the grasper was presented using two parallel rigid plates. It is assumed that the deformation of the grasper is negligible in comparison with the deformation of tissue under the applied contact force.

\subsection{Finite element method}

After creating the 3D model of the kidney and grasper in SolidWorks, the files were imported into Abaqus (DassaultSystèmes, Fr) software. An interaction was defined between the grasper jaws and the kidney (Figure 2(a)). It was assumed that the loading would be directed from the grasper towards the soft tissue. A "general contact" with "finite sliding" was used to describe the interface between the grasper planes and kidney. A "penalty" contact method, with a friction coefficient of 0.15 [9], was chosen to minimize the penetration of the contacting node and elements at the interface. The boundary conditions were applied to the kidney model as zero displacements at the nodes of its top surface, which can present the effect of surrounding tissue. An external loading was applied to the grasper planes to squeeze the kidney tissue at its contact zone. The contact pressure between the grasper planes and the kidney tissue in a $Z$ direction was defined to increase from zero to a maximum of a $0.02 \mathrm{MPa}$ in 2 seconds. As there was a small gap between the grasper jaws and the tissue, a preforce was applied to the jaws to make sure they were in touch with the tissue before applying the original loading. As the geometry of the model was complex, a dynamic explicit procedure was used to solve the FE model. The kidney's model was meshed using 1330 nodes and 6195 elements type C3D4 (Figure 2(b)).

\subsection{Meshless method}

In this study, the Element Free Galerkin (EFG) method was used to simulate the mechanical interactions of the kidney and the grasper. This method presents a high convergence rate and efficiency for models with moving interfaces [10]. The shape functions of the EFG method were constructed using the Moving Least 


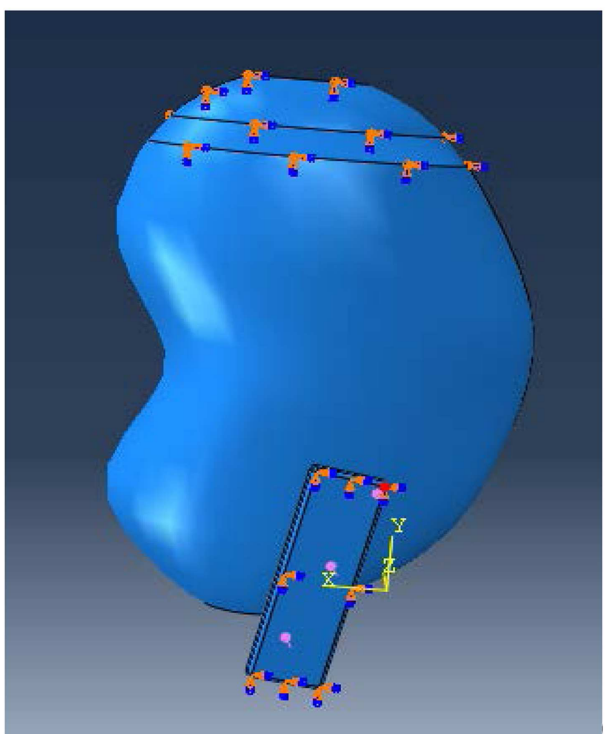

(a)

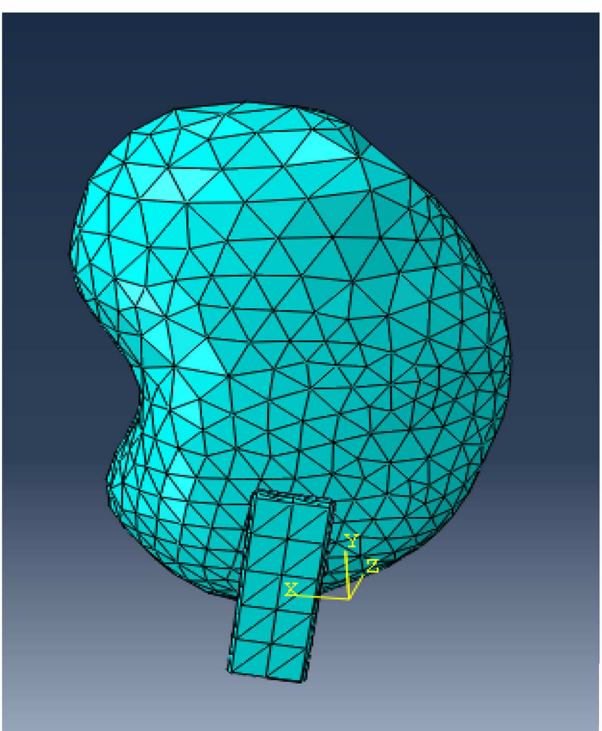

(b)

Figure 2. (a) The 3D geometrical model of the kidney and grasper jaws in Abaqus. (b) The mesh model of the kidney including 1330 nodes and 6195 elements.

Squares (MLS) technique [11]. This approximation introduces $u(x)$ as a function defined in Eq. (1):

$$
u^{h}(x)=\sum_{i=1}^{m} p_{i}(x) a_{i}(x)=p^{T}(x) a(x),
$$

where $p(x)$ are polynomial basis functions, $m$ is the number of basis functions in the column vector, $p(x)$ and $a(x)$ are their coefficients, which are functions of the spatial coordinates, $x$. In the current implementation, a 3D linear basis function was utilized.

The shape functions were derived as below:

$$
\phi(x)=P^{T}(x) A^{-1}(x) B(x),
$$

in which $A$ and $B$ are defined as:

$$
\begin{aligned}
& A(x)=p^{T} w(x) p, \\
& B(x)=W(x) p^{T},
\end{aligned}
$$

and $w(x)$ represents the weight function with a domain of influence.

At the initial step, using the geometry imported from SolidWorks software, the nodes were required to make the general geometry of the kidney. Then, the effective domain was determined for each node, and quadrature cells and Jacobian and weight matrices were generated. A 3D linear basis function and implicit integration were used in the model. The algorithm was implemented in Matlab (Mathworks, US). All boundary conditions and external loadings applied to the model were similar to those of the FE model.

Originally, 83 nodes were implemented in the meshless model and the displacement of these nodes was considered the deformation of the model. But, in order to make a comparison between the meshless and FE models, the displacements of the nodes similar to the FE model were also determined. In order to find the displacements of these points, a meshless solution for the nearest nodes was considered. Using an interpolation procedure, for each node, a new coordinate system was calculated considering its location within the domain. Eq. (5) indicates the formulation used for interpolation in one direction:

$$
d x=\frac{\left(\sum_{i=1}^{n} w_{i} d x_{i}\right)}{\sum_{i=1}^{n} w_{i}}
$$

where $i$ denotes the number of nodes that have the minimum distance from a chosen point, and $w$ represents a coefficient depending upon the distance from the point to each neighboring node.

\section{Results}

\subsection{FE model}

The results of the finite element model and the meshless model for total displacement of the nodal points were post processed using Matlab, as shown in Figure 3. The node coordinates and element specifications were used as input to create the model. The magnitude of the displacement vector for each node was calculated. In Figure 3, the colors show the level of displacement at the model. For instance, the blue areas at the top of the kidney are indicative of minimum displacement, and the orange areas at the bottom of the kidney specify the areas with the largest displacements. 


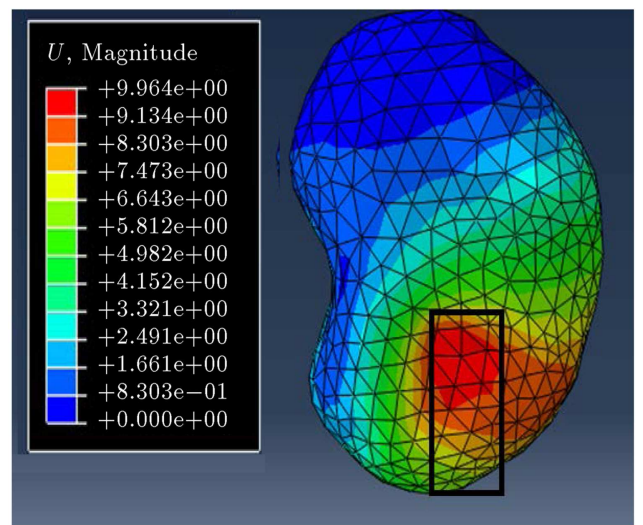

(a)

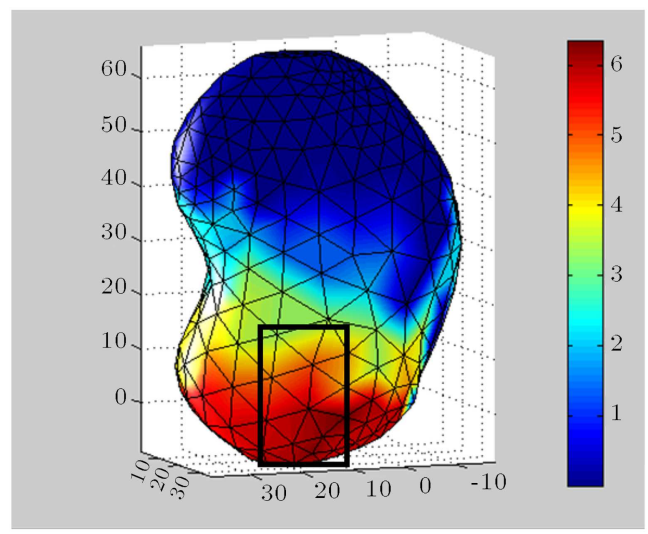

(b)

Figure 3. The 3D kidney deformation: (a) FEM method; and (b) meshless method. Dimensions are in millimeters.

Figure 3(a) shows the results of the finite element method for distribution of the displacements on the kidney. The largest deformation at the kidney occurred at the contact area with the grasper jaw, where the tissue was compressed. The maximum displacement was about $10 \mathrm{~mm}$ in this area. There were also regions of large deformations on the lateral sides of the kidney, near to the contact site, where the tissue was affected by the edges of the graspers. The maximum displacement was about $5 \mathrm{~mm}$ in this area. No displacement was observed at the top of kidney, which was kept fixed in the finite element model. The running time of the FE model, with 1330 nodes and 2621 elements, was about 2 hours on a laptop computer.

\subsection{Meshless model}

The results of the meshless model for the total displacements of 1330 points, equivalent to the nodes of the finite element model, are shown in Figure 3(b). The displacements of these points were found using an interpolation procedure from the meshless solution for the initial 83 nodes. Again, maximum deformations were observed at the contact areas with the grasper jaws, with a maximum displacement of about $7 \mathrm{~mm}$. Also, the large deformation regions, at the lateral sides of the kidney, similar to the FE model, were predicted by the meshless model. A maximum displacement of $3 \mathrm{~mm}$ was observed in this area. No displacement was found at the top of the kidney, due to the boundary conditions applied to the model. The computational time for preprocessing and generating the stiffness and mass matrices of the 83 node meshless model was about 23 seconds. Also, the computational time to solve the meshless model was about 10 seconds, and it took 0.07 seconds to perform the interpolation procedure and find the displacement for the 1330 points.

\subsection{Error analysis}

In order to evaluate the accuracy of the meshless solution, its results should be compared with the FE model using a node by node approach. The displacements obtained from both FEM and meshless methods for all 1330 nodes were compared. The general pattern of the displacement distribution for the two models was in close agreement. There were some differences in the results, which is thought to be due to node scattering, the interpolation procedure and the domain of influence. In addition, the distribution of the nodes in the meshless model was different; fewer nodes in the middle and more nodes at the bottom of the kidney were distributed. Hence, the main differences were found to be at the latter side of the kidney.

Considering $x$ and $y$ as the lateral and distal directions on the kidney's surface, respectively, and $z$ as the direction anterior to the kidney (Figure 1), the differences of the nodal displacements were analyzed at each direction. The maximum displacements predicted by the FE model in $x, y$, and $z$ directions were 5.32 , 2.26 , and 9.79 , respectively. While the corresponding results for meshless model were $0.40,1.55$, and $6.35 \mathrm{~mm}$, respectively.

To verify the accuracy of the results, an error analysis operation was performed. The Root Mean Square (RMS) errors were calculated for all 1330 points in $x, y$ and $z$ directions. The RMS errors are as follows:

$$
\begin{aligned}
& (\mathrm{RMS})_{x}=1.6 \mathrm{~mm}, \\
& (\mathrm{RMS})_{y}=1.0 \mathrm{~mm}, \\
& (\mathrm{RMS})_{z}=2.8 \mathrm{~mm} .
\end{aligned}
$$

\section{Discussion}

In order to use a computer simulation system to train for laparoscopic surgery, a fast and real time computer program is required. This study focuses on developing a biomechanical computer simulator that can evaluate the interactions between a large intra-abdominal organ, i.e. a kidney, and a grasping instrument. Generally, 
undertaking laparoscopic surgery is complex, since the surgery is blind, and manipulation of the tissue is difficult. There is no direct visualization and feedback sensing. Thus, the training models should provide appropriate visual and haptic display in real time.

Previous studies in the literature have often been unable to simulate the grasping procedure of soft organs with high accuracy and feasible computational cost. The finite element model can provide a realistic presentation of soft tissue deformations, but, the finite element procedure is normally slow with high computational cost. Therefore, the finite element method cannot be considered for real time simulations [3].

The meshless method examined in the present study is based on a set of scattered nodes that build the geometry of the soft organ. With the meshless method, during the running process, there is no need to mesh and re-mesh the geometrical model. This would be important when dealing with soft abdominal organs where deformations may be quite large. W With an FE model, re-meshing of the object during the solution procedure may be required, due to the geometrical irregularity of the models.

The results of this study show that the meshless method can provide reasonable accuracy with low computational cost. Close agreement between the results of the meshless model and those of the FEM was observed. The RMS errors for the displacements of the points in the meshless model were within an acceptable range, based on FE references. As a general conclusion, it might be suggested that the meshless method is an efficient computing technique for evaluating the mechanical properties of soft tissue in a real time process.

The results of this study suggest that the differences between the meshless model and FEM results depend upon the node scattering pattern. In general, the high density distribution of nodes was at the contact regions, and the low density distribution of nodes was in other regions to reduce computational time. As a result, higher accuracies were obtained at the regions in contact with the grasper jaws compared to those regions located outside the contact interface. This feature of the meshless method can be used to adjust the accuracy at different areas of the soft tissue model by applying appropriate distribution patterns for the nodes. For instance, at the sensitive areas of the tissue, e.g. near the blood vessels or nerves, higher accuracy can be obtained utilizing a higher density distribution pattern.

Finally, a simple innovative approach was presented in this study to achieve a realistic and comprehensive graphical representation of the deformed organs from the results of the meshless model. Within the current meshless method, only displacement was computed. It is obvious that by increasing the number of nodes, higher computational time would be resulted, which is not the aim of this study. Here, a limited number of nodes was used to solve the meshless model in an acceptable time frame. However, after finding the displacements of the nodes, it is possible to calculate the displacements for a large number of points around the geometrical model by employing an interpolation approach. The algorithm used for the meshless model would be very useful when dealing with irregular, complex and large organ geometries.

\section{Conclusion}

This study suggests that the meshless method is an efficient technique for real time computer modeling of large organs. For the model presented in this study, the computational cost of the meshless method was much less $(99.5 \%$ less $)$ than that of the finite element model.

\section{References}

1. Misra, S., Macura, K.J., Ramesh, K.T. and Okamura, A.M. "The importance of organ geometry and boundary constraints for planning of medical interventions", Medical Engineering and Physics, 31, pp. 195-206 (2009).

2. Cotin, S., Delingette, H. and Ayache, N. "Realtime elastic deformations of soft tissues for surgery simulation", IEEE Transactions on Visualization and Computer Graphics, 5, pp. 62-73 (1999).

3. Tirehdast, M., Mirbagheri, A., Asghari, M. and Farahmand, F. "Modeling of interaction between a threefingered surgical grasper and human spleen", Studies in Health Technology and Informatics, 163, pp. 663669 (2011).

4. Szekely, G., Brechbuhler, Ch., Hutter, R., Rhomberg, A., Ironmonger, N. and Schmid, P. "Modelling of soft tissue deformation for laparoscopic surgery simulation", Medical Image Analysis, 4, pp. 57-66 (2000).

5. Basafa, E. and Farahmand, F. "Real-time simulation of the nonlinear visco-elastic deformations of soft tissues", International Journal of Computer Assisted Radiology and Surgery, 6, pp. 297-307 (2011).

6. Abdi, E., Farahmand, F. and Durali, M. "A meshless EFG-based algorithm for 3D deformable modeling of soft tissue in real-time", Studies in Health Technology and Informatics 2012, 173, pp. 1-7 (2012).

7. Doblare, M., Cueto, E., Calvo, B., Martinez, M., Garcia, J. and Cegonino, J. "On the employ of meshless methods in biomechanics", Computer Methods in Applied Mechanics and Engineering, 194, pp. 801-821 (2005).

8. Tillier, Y., Paccini, A., Durand-Reville, M., Bay, F. and Chenot, J.L. "Three-dimensional finite element modelling for soft tissues surgery", International Congress Series, 1256, pp. 349-355 (2003). 
9. Wu, J.Z., Dong, R.G. and Schopper, A.W. "Analysis of effects of friction on the deformation behavior of soft tissues in unconfined compression tests", Journal of Biomechanics, 37, pp. 147-155 (2004).

10. Guo, X. and Qin, H. "Meshless methods for physicsbased modeling and simulation of deformable models", Science in China, Series F: Information Sciences, 52, pp. 401-417 (2009).

11. Dolbow, J. and Belytschko, T. "An introduction to programming the meshless element free Galerkin method", Archives of Computational Methods in Engineering, 5(3), pp. 207-242 (1998).

\section{Biographies}

Zeinab Saghaei Nooshabadi received her MS degree in BioMechanical Engineering from Sharif University of Technology, Tehran, Iran. Her research interest lies on mechanical and biomedical engineering.

Elahe Abdi received her BS and MS degrees in Mechanical Engineering and Biomechanical Engineering from Sharif University of Technology, Tehran, Iran, in 2009 and 2102, respectively. She is currently at the Institute of Microengineering at EPFL, Switzerland, where she is a doctoral assistant.

Farzam Farahmand received an MS degree in Mechanical Engineering from the University of Tehran, Iran, in 1992, and a PhD degree in Biomechanical Engineering from Imperial College of Science, Technology and Medicine, London, UK, in 1996. He is currently Professor and Head of the Biomechanics Division in the Mechanical Engineering Department of Sharif University of Technology, Tehran, Iran. He also has a joint appointment in the Research Center for Biomedical Technologies and Robotics (RCBTR) in Tehran University of Medical Sciences, Tehran, Iran, where he is head of the Medical Robotics Group. His research is focused on human motion, orthopedic biomechanics and medical robotics. In his career, he has developed several medical instruments and devices and published numerous journal and conference papers in different fields of biomechanics and biomedical robotics

Roya Narimani received her BS degree in Electrical and Electronics Engineering from California State University, Sacramento, USA, in 1980, and her MS degree in Bioengineering from the University of Utah, USA, in 1982. She is currently an instructor in the Mechanical Engineering Department of Sharif University of Technology, and Director of the Applied Electronics Laboratory. Her research is focused on bioinstrumentation and rehabilitation.

Mahmoud Chizari received MSc degree in Mechanical Engineering from UMIST, UK, and PhD degree in Bioengineering from The University of Manchester, UK, in 2005 and 2008, respectively. He completed his PostPhD in Biosurgical Engineering with the University of Aberdeen, UK, in 2011. He is currently member of the Orthopaedic Research and Learning Centre, where he is a lecturer in the Department of Mechanical, Aerospace and Civil Engineering at Brunel University, London, UK. He is also Adjunct Professor in the Mechanical Engineering Department of Sharif University of Technology, Tehran, Iran. 